\title{
Circuit-seq: Circular reconstruction of cut in vitro transposed plasmids using Nanopore sequencing
}

\author{
Francesco E. Emiliani ${ }^{1^{*}}$, lan Hsu ${ }^{1}$, and Aaron McKenna ${ }^{12^{*}}$ \\ ${ }^{1}$ Department of Molecular and Systems Biology, Geisel School of Medicine, Dartmouth College, \\ Lebanon, NH 03756, USA \\ ${ }^{2}$ Norris Cotton Cancer Center, Dartmouth-Hitchcock Medical Center, Lebanon, NH 03756, USA \\ * To whom correspondence should be addressed \\ E-mail: Francesco.E.Emiliani.GR@Dartmouth.edu, McKenna.Aaron@gmail.com
}

\begin{abstract}
Recombinant DNA is a fundamental tool in biotechnology and medicine. Validation of the resulting plasmid sequence is a critical and time-consuming step, which has been dominated for the last 35 years by Sanger sequencing. As plasmid sequences grow more complex with new DNA synthesis and cloning techniques, we need new approaches that address the corresponding validation challenges at scale. Here we prototype a highthroughput plasmid sequencing approach using DNA transposition and Oxford Nanopore sequencing. Our method, Circuit-seq, creates robust, full-length, and accurate plasmid assemblies without prior knowledge of the underlying sequence for approximately $\$ 1.50$ per plasmid. We demonstrate the power of Circuit-seq across a wide range of plasmid sizes and complexities, generating accurate and contiguous plasmid maps. We then leverage our long read-data to characterize epigenetic marks and estimate plasmid contamination levels. Circuit-seq scales to large numbers of samples at a lower cost than commercial Sanger sequencing, accelerating a key step in synthetic biology, with low startup costs make it practical for individual laboratories.
\end{abstract}

Keywords: Long-reads, plasmid, cloning, assembly, sequencing

\section{Introduction}

Plasmids are the core building block of recombinant DNA. Researchers can combine and synthesize novel DNA sequences to manipulate cellular biology: from emerging therapeutics like CAR-T cells to synthetic biology circuits that perturb cellular functions (Antebi et al. 2017; Hernandez-Lopez et al. 2021). These constructs are often sensitive to imperfections and require careful sequence validation. Plasmid sequence is typically verified using chain-termination sequencing (also known as Sanger sequencing), first described in 1977 (Sanger, Nicklen, and Coulson 1977). Sanger sequencing requires a complementary oligonucleotide to bind upstream of the sequence of interest, from which random terminated fragments are used to create a consensus sequence (Heather and Chain 2016).
However, plasmid sequences are now routinely greater than 10 kilobases, requiring a large number of custom primers, and repetitive regions or imbalanced nucleotide content can be especially challenging for Sanger sequencing (Kieleczawa, Dunn, and Studier 1992). To circumvent these limitations, the field has developed a number of secondary techniques to validate the entirety of the sequence, including restriction enzyme mapping or successive primer walking. The collective reagents and time required to validate individual sequences can halt progress or limit the scope of scientific questions; as we incorporate advances in DNA construction and decreased DNA synthesis costs, we expect these challenges of scale and accuracy to only grow more acute (Gibson et al. 2009; Hughes and Ellington 2017).

Our lab's need to validate complex plasmid assemblies at scale led us to assess the available methods for high- 
A

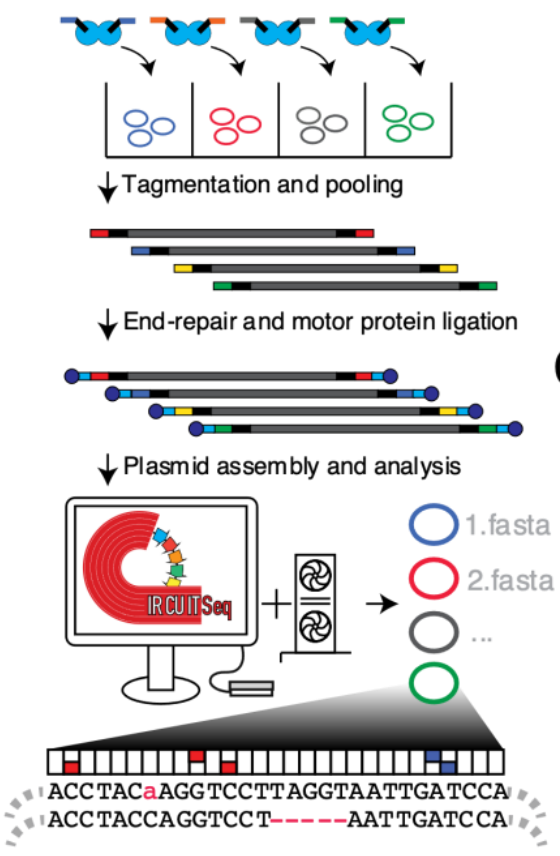

B

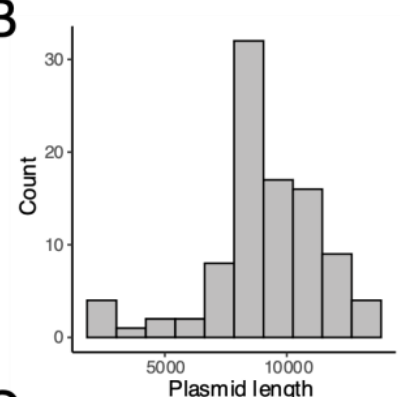

$\mathrm{C}$

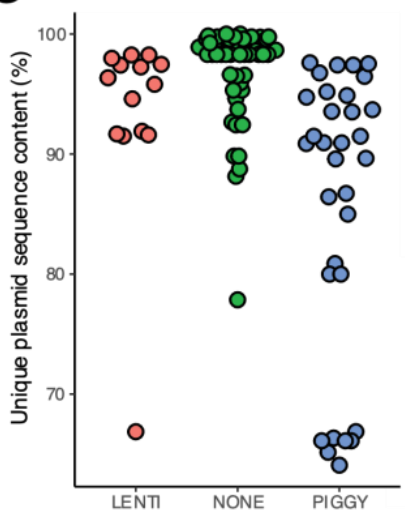

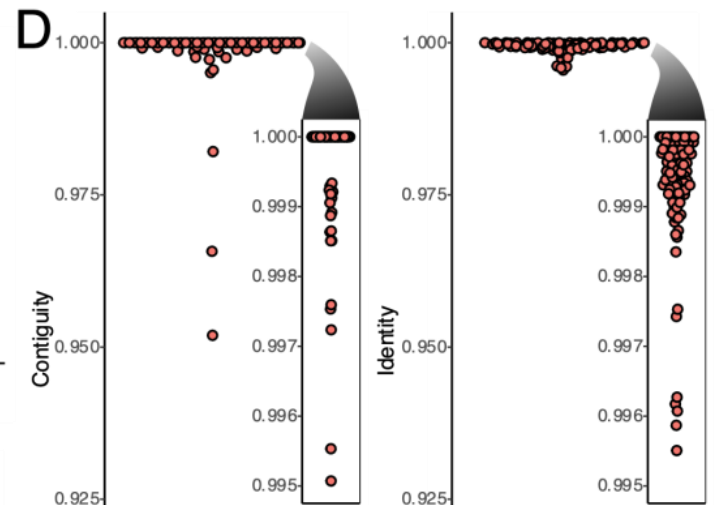
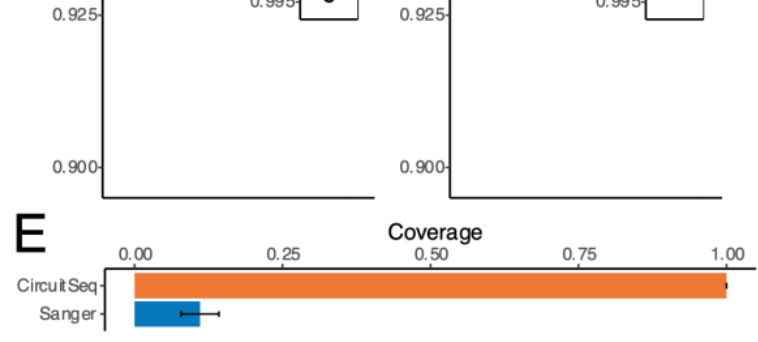

F
$\begin{array}{r}\text { Circuitseq } \\ \text { Sanger }\end{array}$

Figure 1. (A). Schematic representation of the Circuit-seq. Plasmids are arranged in a 96 well are tagmented with well-specific barcodes. Samples are then pooled, end-repaired, adapter-ligated, and processed on an Oxford Nanopore Flongle flowcell. The generated data is then run through a custom NextFlow pipeline producing the final assembled sequences. (B). A distribution of the plasmid sizes used in our experiment. (C). Unique (non-repetitive) plasmid rates for our input pool. Lentiviral (LENTI) and piggybac (PIGGY) payload plasmids have more repetitive sequence in their backbones, a key criteria to challenge our assembly pipeline. (D). Contiguity and identity scores for the polished assemblies were calculated by comparison to the known reference. (E). Proportion of the full plasmid covered by Circuit-seq assemblies and Sanger sequencing. (F). The error rate for Circuit-seq assemblies and Sanger sequencing was calculated by comparison to known reference. Sanger sequences were pre-filtered to remove sequences with $>10 \%$ error to exclude technical errors from the analysis.

throughput sequencing and assembly. Recent work has demonstrated the utility of high-throughput plasmid verification using Illumina sequencing technologies (Shapland et al. 2015; Gallegos et al. 2020). The result has been impressive: Gallegos et al. were able to fully assemble a 96-well plate of 2.5 to $3.3 \mathrm{~kb}$ plasmids. Unfortunately, these short-read approaches often struggle to achieve high contiguity for more complex plasmids: the same pipeline in Gallegos et al. was only able to assemble $25 \%$ of a more complex plasmid pool containing longer repeat elements (Gallegos et al. 2020; Shapland et al. 2015). Sequencing cost is also an issue, as large numbers of plasmids must be pooled to overcome reagent costs, and upfront machine costs are often prohibitive for labs that don't have direct access to an Illumina machine (Gallegos et al. 2020).

Inspired by a recent publication of a PCR-based plasmid sequencing method (Currin et al. 2019) and the low-cost Nanopore Flongle flowcell, we turned our attention to the Oxford Nanopore long-read sequencing platform. We reasoned that long-read sequences could overcome challenging repeat regions, especially if we were able to preserve the contiguity of the underlying sequence through library generation. This key advantage, coupled with recent advances in sequencing output and computational methods improvement could allow us to accurately assemble even the most complex plasmids. Here we detail our approach, Circuit-seq (Circular reconstruction of $\underline{\text { cut }}$ in vitro transposed plasmids), which generates complete maps of synthetic DNA constructs within 24 hours at a cost that is competitive with single chain-terminating sequencing reactions. These assemblies can then be annotated with epigenetic base modifications, and our long-read data can be used to estimate input contamination levels, providing a comprehensive sequence characterization for a wide variety of downstream applications. A Nextflow pipeline is also available implementing the computational aspects of Circuit-seq: https://github.com/mckennalab/Circuitseg (Di Tommaso et al. 2017). 


\section{Results}

\section{Tagmentation leads to robust multiplexing of a diverse set of 96 plasmids}

Multiplexing approaches for sequencing have increased throughput and decreased per-sample costs (Shendure et al. 2017). Thus, we set out to develop an approach that multiplexed the capture and assembly of full-length plasmid sequences on a long-read sequencing platform. We initially considered two library generation approaches: (1) restriction digestion of plasmids followed by barcode ligation (fig. s1) and (2) barcode tagmentation with the Tn5 transposase (fig. 1a) (Reznikoff 2008). While both approaches proved successful, we decided to employ the sequenceagnostic Tn5 transposition technique.

The Tn5 enzyme is commonly used for transposasemediated adaptor insertion and subsequent sequencing (A. Adey et al. 2010). Tn5 is loaded in vitro with an oligonucleotide (oligo) containing the Tn5 mosaic sequence (Reznikoff 2008; A. Adey et al. 2010; A. C. Adey 2021). To uniquely tag individual plasmids, we extended these 19bp mosaic sequences with a set of 96 error-resistant $17 \mathrm{bp}$ barcodes from Hawkins et al. (fig. 1a, table s3) (Hawkins et al. 2018). Our goal was a limited tagmentation of each plasmid to preserve contiguity and to lower the per-reaction cost. We used limiting quantities of oligonucleotides in an excess of Tn5 to restrict tagmentation, which we tested over a range of concentrations, near the lowest levels used by Picelli et al. (Picelli et al. 2014). Tn5 tagmented libraries were then pooled and prepared using the Oxford Nanopore ligation sequencing kit and loaded onto an individual Flongle flow cell. The results of our optimization (fig. s2a) show that our approach is robust over the tested conditions, generating a sizable proportion of full-length reads. Increasing the amount of $\mathrm{Tn} 5$ or the duration of tagmentation increased the number of tagmentations per plasmid, though we recovered sufficient fragments from all conditions to generate correct assemblies (fig. s2b).

We next tagmented a diverse set of 96 plasmids using the optimized conditions above. Each input plasmid has a known sequence map, ranging in size from 2,433 to 13,286 basepairs (fig. 1b). The input plasmids had a wide range of repetitive sequence content (ranging from 0 to $36 \%$ of the known map) incorporating both lentiviral and PiggyBac integration vectors (fig. 1c). We also used an improved set of Tn5 sample barcodes (v2) with increased hamming distance to improve barcode recovery (table s4). We then demultiplexed and processed individual plasmids with a custom NextFlow pipeline (Di Tommaso et al. 2017). Reads assigned to plasmid barcode IDs accounted for $81 \%$ of reads, resulting in 150-4619x (median 1118) coverage of our plasmid set, with little correlation to input plasmid length $(r=-0.16$, fig. s3a). As expected, we observed peaks in fragment sizes at known plasmid lengths, with increasing proportions of smaller fragments which are presumably from DNA fragmentation in library preparation and sequencer length bias (fig. s3b) (Amarasinghe et al. 2020). Consistent with this hypothesis, we observe more full-length fragments in smaller plasmids (fig. s3c). We then filtered and corrected each sample's reads to generate errorcorrected consensus sequences where the distribution more closely matches single and double tagmentation events (fig. s3d) (Koren et al. 2017).

\section{De novo plasmid assembly and validation}

We then assembled plasmids de novo from the corrected reads, avoiding any biases from referencebased assembly approaches (Lunter and Goodson 2011). We used a two-pronged assembly approach, leveraging both Flye and Miniasm long-read assemblers to generate competing initial consensuses from which the longest single-contig assembly is taken forward ( $R$. R. Wick and Holt 2019; Kolmogorov et al., n.d.) (https://github.com//h3/miniasm). This is followed by subsequent rounds of refinement with Medaka and custom post-processing steps to eliminate whole genome duplications and other common assembly artifacts (https://github.com/nanoporetech/medaka). Our pipeline was able to assemble 95 of 96 plasmids to a $100.00 \%$ median contiguity and $99.95 \%$ identity (fig. 1d), an improvement over existing Illumina sequencing approaches (Gallegos et al. 2020). No reads for the missing assembly were recovered from the raw data, suggesting that it was due to technical error during sample preparation.

We next wanted to determine the accuracy of our approach compared to conventional validation techniques. We collected 64 Sanger sequencing reactions from 36 different plasmids within our Nanopore assembly dataset. We converted the resulting trace files and filtered them by quality score using Mott's algorithm to clip low-quality regions (Ewing et al. 1998). We further removed seven Sanger sequencing results where the error rate was above $10 \%$ to avoid biasing our results from potential user error (fig. s4e and f, methods). The 57 remaining Sanger sequencing results covered a median of $10.3 \%$ of their respective plasmid with an average aligned length of 1023 bases (fig. 1e). The 
Sanger median per-base error rate was $0.4 \%$, approximately a 10 -fold higher rate than our Nanopore assembly approach (fig. 1f). In aggregate, our pipeline successfully assembled high contiguity and identity maps even when faced with highly repetitive sequences.

\section{Plasmid epigenetics}

Given the success of our assembly pipeline, we wondered if we could use our rich sequencing data to provide validation that is inaccessible from chainterminating sequencing results. Bacterial epigenetic patterns are carried over to plasmid sequences; both $\mathrm{dcm}$ and dam methylation can be transferred to

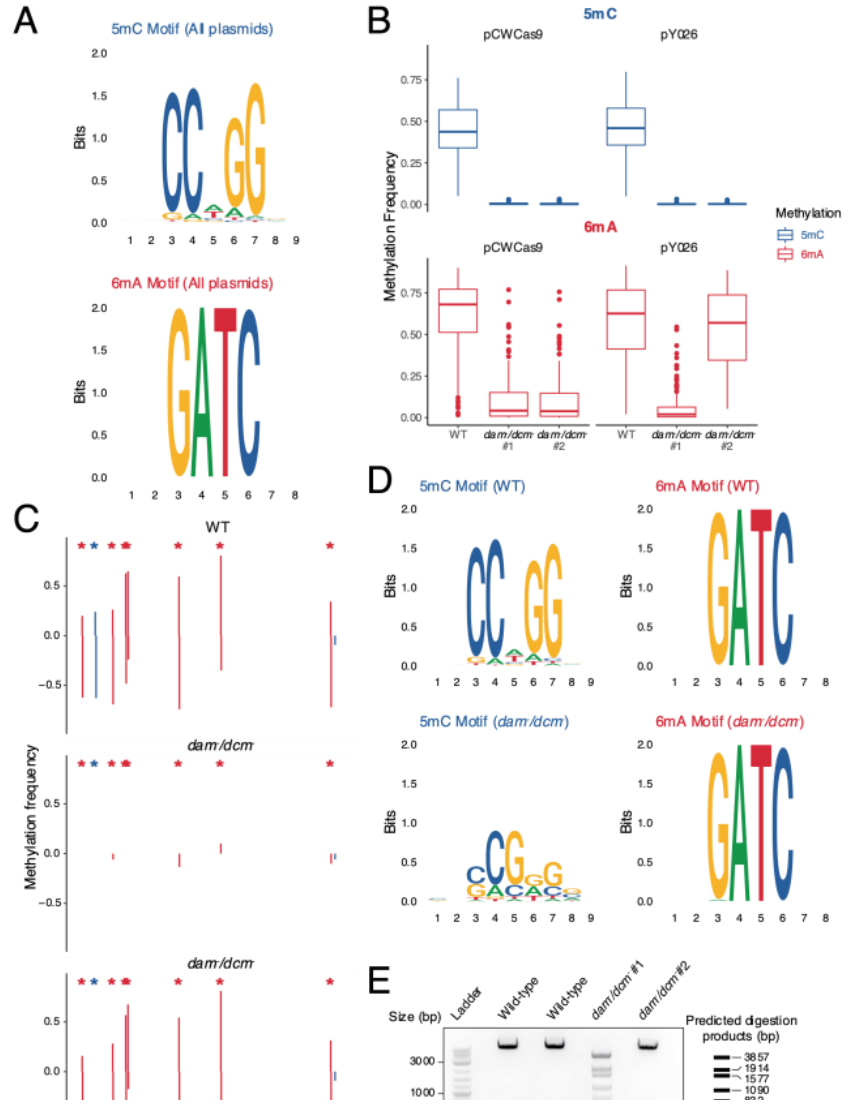

Figure 2. (A) DNA sequence logos generated from plasmid methylated 5-methylcytosine $(5 \mathrm{mC})$ and 6-methyladenine $(6 \mathrm{~mA})$ call sequences representing known $\mathrm{dcm}$ and dam consensus sequences, respectively. (B) Global $5 \mathrm{mC}$ and $6 \mathrm{~mA}$ rates from plasmids grown in conventional $\mathrm{dcm}^{+} / \mathrm{dam}^{+}$E. coli (WT) and $\mathrm{dcm}^{-} / \mathrm{dam}^{-}$E. coli (methods). (C) Visualizing methylation frequencies across a region of pY026 plasmid shows methylation localizing to consensus motifs (asterisks) in pairs, corresponding to the top and bottom strands. And highlights the complete loss of $5 \mathrm{mC}$ methylation in the $\mathrm{dcm}^{-} / \mathrm{dam}^{-}$ samples, and the partial and complete conversion to $\mathrm{dam}^{+}$in the first and second $\mathrm{dcm} / \mathrm{dam}$ samples, respectively. (D) DNA sequence logo plots from methylation sites of plasmids grown in WT and $\mathrm{dcm}^{-} / \mathrm{dam}^{-}$ $E$. coli showing the loss of $5 \mathrm{mC}$ but recovery of $6 \mathrm{~mA}$. (E) Validation of methylation using $6 \mathrm{~mA}$ sensitive Bcll digestion of Mlul linearized pY026 plasmid. Bcll digestion failed to cleave the methylated plasmid as well as the $\mathrm{dam}^{+}$recovered plasmid, but near-complete digestion of the dam plasmid. Predicted digestion products annotated on the right. matching recognition sites in plasmids propagated by $E$. coli (Marinus and Løbner-Olesen 2014). This methylation has a number of implications for synthetic biology, including methylation-sensitive restriction enzymes, the stability of repeat sequences, and downstream sensitivity issues in the target organism (Kolek et al. 2016; Nichol and Pearson 2002). Given Oxford Nanopore's ability to directly capture DNA modifications, we were interested to see if we could profile the epigenetic status of our assemblies. We then extended our pipeline to call methylation modifications present in each plasmid assembly. As initial validation of this approach, we created consensus DNA sequence logos from the 6 methyladenine $(6 \mathrm{~mA})$ and 5 methylcytosine $(5 \mathrm{mC})$ patterns detected in our 96plasmid run, recovering known sequences of dam (GATC) and dcm (CCYGG) (fig. 2a).

To further validate the accuracy of our approach we then transformed plasmids into both NEB Stable Competent cells (WT) and $\mathrm{dam}^{-} / \mathrm{dcm}^{\mathrm{c}}$ competent cells that lack both methyltransferases. One WT plasmid and two separate colonies of $\mathrm{dam}^{-} / \mathrm{dcm}^{-}$plasmids were processed according to our normal Circuit-seq protocol. In the dam $1 \mathrm{dcm}$ plasmids, methylation calls at motif sequences show a drastic drop of $6 \mathrm{~mA}$ and a near-complete loss of $5 \mathrm{mC}$ (fig. 2b). The low, but not abolished, levels of $6 \mathrm{~mA}$ can be explained by spontaneous re-activation of dam through the loss of the knockout transposon sequence (New England Biolabs n.d.). This is evident in the second replicate of pY026 dam/ $/ \mathrm{dcm}^{-}$which has a nearcomplete reactivation of dam but a complete absence of $\mathrm{dcm}$ activity. Highlighting a stretch of this pY026 assembly, we see a mirroring of methylation patterns on each of the positive and negative strands, confirming that the regained dam activity is consistent with true methylation patterns (fig. 2c). As can also be seen in the DNA sequence motifs from the various conditions, there was no clear sequence motif enrichment in the $5 \mathrm{mC}$ motif in the dam $/ \mathrm{dcm}^{-}$samples, while the $6 \mathrm{~mA}$ successfully recreates the dam motif (fig. 2d). Finally, to experimentally validate the methylation levels, we digested plasmids with a dam-sensitive restriction enzyme, Bcll. The resulting gel shows near-complete digestion of the first $d a m^{-} / \mathrm{dcm}^{-}$plasmid but no visible fragments in the second colony, validating the computational characterization (fig. 2e).

\section{Contamination detection}

Plasmid preparations can be contaminated by bacterial DNA or other passenger plasmids that confound downstream experiments. Given the number of sequencing reads produced for each plasmid, we 
A

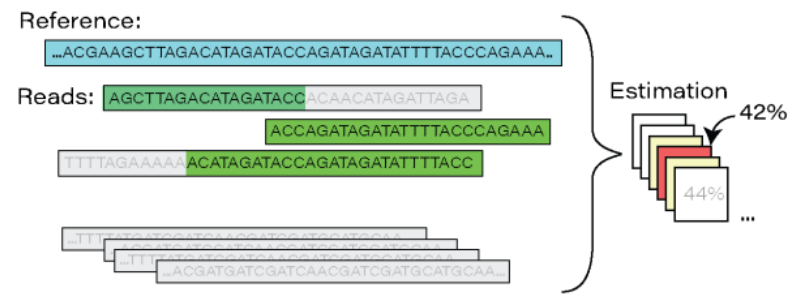

B
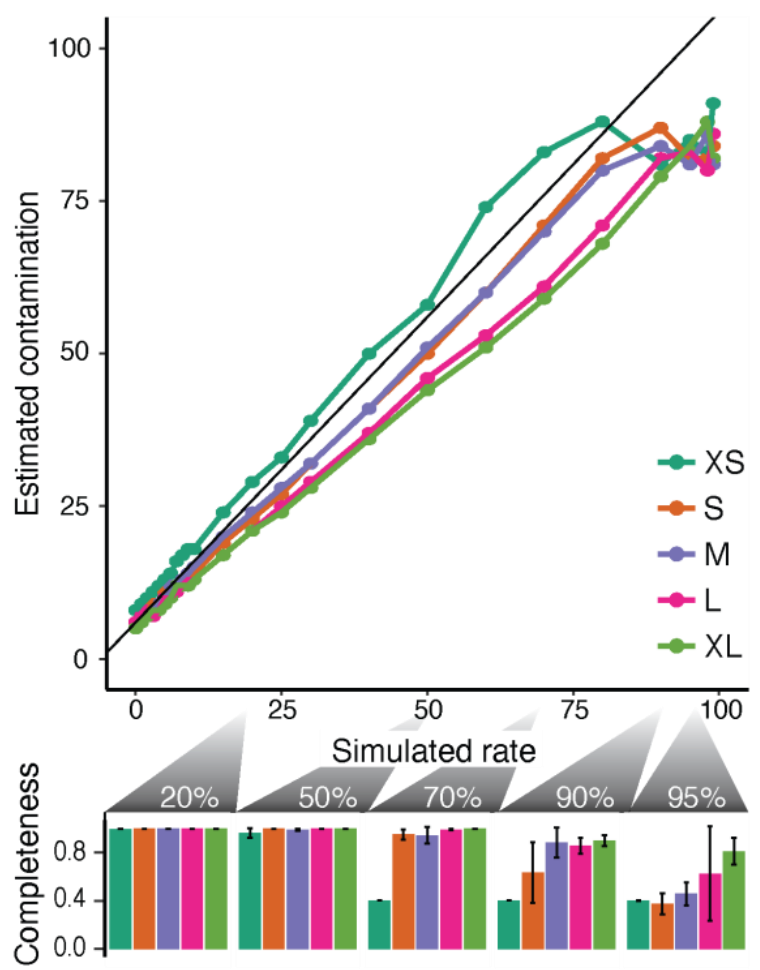

C

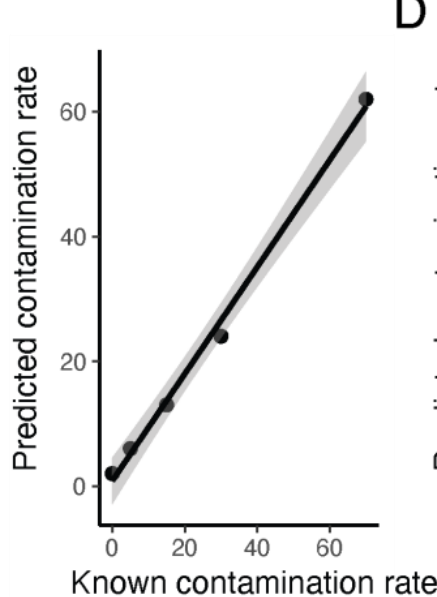

D

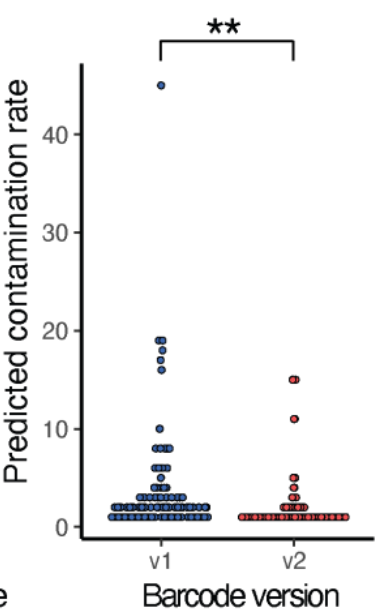

wondered if we could leverage this read depth to estimate potential contamination levels. Given the shared components of many plasmids, i.e. the origin of replication or lentivirus components, we reasoned that previously developed SNV-based contamination estimation methods would be ill-suited for the task (Cibulskis et al. 2011; Jun et al. 2012). Instead, we used a simple Bayesian model to leverage the proportion of
Figure 3. (A) Contamination rates are calculated from the proportion of unaligned bases and the global shared sequence proportions between plasmids, estimated over a range of contamination hypotheses (methods). (B) In silico contamination estimates of assembled Addgene plasmids using simulated reads over a range of plasmid lengths (upper panel, $X S=e x t r a$ small to $X L=e x t r a$ large maps). Our estimates track the programmed rates well $(r=0.97$ RSME = 9.1), though at higher contamination levels our assemblies had issues that result in poor contamination estimates, seen in the assembly completeness drop off for small plasmids at $>70 \%$ contamination and at $>90 \%$ for larger plasmids (lower panel). (C) Correlation of an in vitro mixing experiment of two known plasmids prior to sequencing and assembly. Contamination estimates were well correlated to their mixing proportion $(r=0.997, \mathrm{RSME}=4.66)$ though we failed to assemble the plasmids at the two highest contamination levels (85 and 95\% contaminated). (D) Estimated contamination rates significantly dropped with our improved Tn5 barcoding design ( $p=$ 0.00119).

unaligned bases ('clipped' bases) as well as unmapped reads during sequence alignment, taking into account the shared nature of plasmid components (fig. 3a, methods).

We first validated our approach using computationally generated reads from commonly used plasmids available from the Addgene repository. Five plasmids were chosen spanning a range of sizes and were subsequently contaminated by random reads from 91 other constructs at set levels. The simulated reads were then used as input to our existing pipeline and our computational tool was used to predict contamination levels (fig. $\mathbf{3 b}$, top). Contamination levels were relatively well-predicted by our simple method $(r=0.97$, RSME = 9.1), though were less accurate at high contamination levels due to underlying assembly failures (fig. $\mathbf{3 b}$, bottom, fig. s4a). When we estimated contamination using known plasmid maps instead of our assemblies, we saw similar results, with more consistent estimates at higher contamination levels $(r=0.99$, RSME $=7.2$, fig. s4b).

To further validate our contamination pipeline, we experimentally mixed one plasmid preparation into another over a range of contamination rates. We then sequenced these mixtures in individual wells in an additional Circuit-seq run. Our two most contaminated plasmids, $85 \%$ and $95 \%$ contaminated, failed to assemble, but computational estimates in the remaining samples tracked the known experimental rates $(r=$ 0.997, RSME $=4.66$ ) (fig. 3c). We then profiled overall contamination rates in both our v1 and $\mathrm{v} 2$ barcoding runs (fig. 3d). Contamination levels were correlated with assembly errors (Pearson's $r=0.32$ ), suggesting an avenue to further improve assembly results.

\section{Discussion}

Here we detail our new high-throughput technique, Circuit-seq, an end-to-end plasmid validation pipeline that leads to near-perfect assemblies. This technique, 
complementing other 2nd and 3rd generation sequencing approaches, provides a comprehensive map of both simple and complex plasmids. In comparison to existing Illumina-based techniques, our long-read approach can assemble through large repetitive regions, as well as to characterize epigenetic marks leveraging the unique advantages of the Nanopore platform. We are then able to couple this with computational tools to provide end-users a comprehensive view of the resulting plasmid with errors rates an order of magnitude lower than Sanger sequencing.

Our assemblies are also cost-effective. The reagents required per 96-well run are approximately $\$ 140$ (or < $\$ 1.50$ per plasmid), significantly cheaper than commercial Sanger sequencing (table s1). Additionally, the low start-up cost of the Oxford Nanopore platform allows researchers with even moderate validation needs to consider this approach. Higher density barcoding (for instance 384-well plates in parallel) would further reduce costs. Additionally, Circuit-seq can be run with the larger Minion flow cells that produce data at 5-10x the speed, reducing the amount of time required to obtain assemblies. The Circuit-seq protocol is relatively straightforward once the reagents are obtained, and new users in our lab have successfully generated data in their initial experiment. To further enable the adoption of this technique, we've made all barcode sequences and computational tools publicly available.

In the future, we hope to adopt new computational and experimental improvements on the Oxford Nanopore platform into Circuit-seq, including higher accuracy Nanopore kits and flowcells. It would be straightforward to include these improvements as they become available for the lower-output Flongle, further decreasing error rates and potentially solving our limited remaining contiguity challenges. We have also had preliminary success directly sequencing plasmids from bacterial colonies, replacing traditional colony PCR, though optimization is still needed to increase consistency and yield.

We have found Circuit-seq to be of immense practical value. This is especially true for projects with highly repetitive or complex sequences, where Sanger sequencing results were hard to interpret or outright failed. Even in routine experiments, the effort of Circuitseq is rewarded when we discover a plasmid backbone mutation or mislabeled tube that would have gone undiagnosed until much later in the project. Combined with the competitive cost, we are excited by the prospect of Circuit-seq or other modern techniques supplanting
Sanger sequencing, providing a single-pass, comprehensive validation of DNA constructs.

\section{Acknowledgements}

We would like to thank the members of the McKenna lab for experimental help, advice, and for providing the stream of complex plasmids needed to validate this approach. An additional thanks to the members of the Dartmouth community who also contributed plasmids in the early development phase. We would especially like to thank Rachel Saxe and Maryam Fathi for testing both the protocol and computational pipelines. This work was supported by funding from the Neukom Insitute at Dartmouth College and The Norris Cotton Cancer Center (NCI 5P30CA023108-37). A.M. is supported by $\mathrm{NIH/NHGRI} \mathrm{(R00} \mathrm{HG010152-04),} \mathrm{the} \mathrm{Pew} \mathrm{Biomedical}$ Scholars Fellowship, and the V Foundation. F.E. was supported from the Tenney Fellowship and I.H. from the Neukom Insitute and the Sophomore Research Scholarship at Dartmouth College.

\section{Methods}

\section{Restriction digest approach}

We created six test ligation adapters with unique 20 basepair barcode identifiers (table s2), containing Xhol, EcoRI, Xbal, Bmtl, Bcll, and Sacl restriction sites. Restriction sites were chosen by scanning a listing of all Addgene plasmid sequences, removing sequences shorter than 2000 bases, and generating a cover-set of enzymes that would maximize the number of sequences with at least one single or double-cut enzyme location (fig. s1). A common forward and a unique reverse adapters were annealed at equal molar ratio and extended with a single cycle of Kapa HiFi polyermase using the manufacturer recommended conditions and an extension time of 5 minutes. Adapters were then cut with the target restruction enzyme and purified with Zymo Research Clean \& Concentrate kit. Target plasmids were processed similarly in parallel. Adapters were then ligated to their corresponding plasmid at $3 x$ molar excess using New England Biolab's Quick Ligation kit for 10 minutes at room temperature, followed by a $0.5 \mathrm{X}$ Ampure cleanup to remove excess Adapters, followed by standard Oxford Nanopore ONT LSK-109 or ONT LSK-110 ligation protocols and loaded onto a Oxford Nanopore Flongle flowcell.

\section{Tn5 barcode design}

Our 17-nucleotide (nt) barcodes were obtained from the Hawkins et al. using a set with a minimum of 2-error corrections (Hawkins et al. 2018)(table s3). For our version 2 barcodes (v2) we used 27nt barcode 
sequences, combing Finkelstein's 13nt and 14nt with 2 error corrections were randomly sampled from the Finkelstein et al. sets, combined, and analyzed using the $R$ package DNABarcodes. We resampled 10,000 combinations and selected an optimized set with a median hamming distance of 30 and a guaranteed error correction of 7bp (Buschmann and Bystrykh 2013). The resulting primers were then screened with Integrated DNA Technologies (IDT) OligoAnalyzer to select 96 primers with optimal delta $\mathrm{G}>-4 \mathrm{kcal} / \mathrm{mole}$ (table s4). Barcodes were annealed with a common phosphorylated oligo (/5Phos/CTGTCTCTTATACACATCT) by heating to $95^{\circ} \mathrm{C}$ for 5 minutes and slowly cooling to room temperature.

Tn5 purification and storage

Tn5 was purified by the University of California Berkeley Quantitative Bioscience MacroLab Core Facility as per previously described protocols (Hennig et al. 2018). A long-term storage stock solution of Tn5 at $4 \mathrm{mg} / \mathrm{mL}$ was kept at $-80^{\circ} \mathrm{C}$ in storage buffer $(50 \mathrm{mM}$ Tris- $\mathrm{HCl} \mathrm{pH} 7.5$, $800 \mathrm{mM} \mathrm{NaCl}, 0.2$ mM EDTA, 2 mM DTT, 10\% glycerol). A stock solution at $40 \mathrm{ug} / \mathrm{mL} \mathrm{Tn} 5$ was kept at $-20^{\circ} \mathrm{C}$ in the working buffer $(50 \mathrm{mM}$ Tris- $\mathrm{HCl} \mathrm{pH} 7.5,800 \mathrm{mM} \mathrm{NaCl}$, 0.2 mM EDTA, 2 mM DTT, 50\% glycerol).

Tagmentation reactions

For 96 plasmid on an r9.4 Flongle: 50ng of plasmid was combined with 2.5pmoles of annealed barcode oligo and $40 \mathrm{ng}$ of $\mathrm{Tn} 5$ in reaction buffer $(50 \mathrm{mM}$ Tris-acetate $\mathrm{pH}$ $7.5,150 \mathrm{mM}$ potassium acetate, $10 \mathrm{mM}$ magnesium acetate, $4 \mathrm{mM}$ spermidine, $1 \mathrm{mM}$ DTT) in a 5 ul reaction. For runs with fewer than 48 plasmids, all quantities can be doubled. Using a thermocycler, samples were incubated at $23^{\circ} \mathrm{C}$ for 10 minutes, $37^{\circ} \mathrm{C}$ for 10 minutes, and then $55^{\circ} \mathrm{C}$ for 5 minutes. The reaction was stopped with 1 volume of $0.2 \%$ SDS for 5 minutes at $23^{\circ} \mathrm{C}$. Samples were pooled and cleaned up with $0.5 x$ volume of Ampure beads according to established protocols and eluted in $25 \mathrm{ul}$ of water. For the $\mathrm{r} 10.3$ minion run all volumes were doubled but the rest of the protocol remained the same.

\section{Oxford Nanopore (ONT) library preparation}

Plasmids sequences are available for our V1 and V2 efforts in supplemental tables 5 and 6 . Samples were prepared in accordance with the ONT LSK-110 manual for Flongle libraries. In brief, the purified tagmented libraries were repaired using a combined NEBNext FFPE DNA Repair Mix and NEBNext Ultra II End Repair/dA-Tailing Module by incubating at $20^{\circ} \mathrm{C}$ for 7.5 minutes and $65^{\circ} \mathrm{C}$ for 7.5 minutes. Samples were purified with $0.5 \mathrm{x}$ vol of Ampure beads and eluted into 30ul of water. Ligation was performed with NEB Quick
Ligase, ONT ligation buffer, and Oxford Nanopore's adapter mix (AMX) for 10 minutes at room temperature. Samples were purified with $0.5 x$ Ampure beads and then washed with ONT long fragment wash buffer instead of $70 \%$ ethanol, and eluted into 7 ul of ONT elution buffer. $5 \mathrm{ul}$ of the resulting library was loaded into a Flongle as per ONT specifications.

\section{Demethylated plasmid preparation}

Plasmids were transformed into NEB dam-/dcmcompetent $E$. coli (C2925I) as per the vendor's protocols and plated onto LB-agar with ampicillin. The following day we picked two colonies per plasmid and grew overnight in LB-Broth with ampicillin for plasmid extraction with the Qiagen Miniprep kit.

\section{Data Generation and Assessment Workflow}

We established a NextFlow pipeline using a prepackaged Docker container to facilitate adoption and reproducibility (Di Tommaso et al. 2017). This pipeline performs all computational steps from base calling to assembly polishing with the ability to create assembly statistics if reference sequences are provided. The pipeline and related documentation are available on our Github (https://github.com/mckennalab/Circuitseq).

Basecalling was performed with ONT guppy software version $5.0 .16+b 9 f c d 7 b 5 b$ using the r941_min_sup_g507 mode. When necessary the pipeline parameter file can be modified to work with different basecalling models or flow cells. Fastq files are then binned using Oxford Nanopore's Guppy demultiplexing function using a custom barcode configuration file. Porechop (Pryszcz and Novoa 2021; R. Wick et al. 2017)(https://github.com/rrwick/Porechop) is then used to trim adaptor sequences and discard chimeric reads resulting from aberrant ligation products. Sequences shorter than 500bp are filtered with nanofilt (De Coster et al. 2018)(https://github.com/wdecoster/nanofilt). Filtered reads are corrected with Canu (Koren et al. 2017)(https://github.com/marbl/canu) and assembled with miniasm (https://github.com/lh3/miniasm) and Flye assemblers (Kolmogorov et al., n.d.). The assembly is then polished with one round of Medaka (https://github.com/nanoporetech/medaka) to reduce error, which increases the fidelity of duplication removal using DupScoop (https://github.com/mckennalab/DupScoop), a tool we developed to resolve a common error in Flye assemblies where the assembly is perfectly duplicated. The assemblies are then rotated $50 \%$ of their length. We found that the edges of the circular assemblies do not get polished as effectively, and rotating the assemblies allows these sequences that are now in the center of the assembly to be polished efficiently during the following three rounds of Medaka. If a reference is provided, the 
assemblies undergo assessment of identity and contiguity (R. R. Wick and Holt 2019), which generate the values used within the paper. We then define each assembly's 'completeness', ranging from $[0,1]$, as the value:

$(1-\operatorname{abs}(1-$ contiguity $)) *(1-a b s(1-i d e n t i t y))$

This is a somewhat conservative calculation, given that contiguity and identity values are not fully independent.

\section{Methylation calling and analyisis}

The raw fast5 reads are demultiplexed using the Guppy demultiplexed fastq files using fast5_subset from ont_fast5_api

(https://github.com/nanoporetech/ont fast5 api). The fast5s are then basecalled using Guppy version $4.4 .2+9623 c 16$ with the methylation-trained model dna_r9.4.1_450bps_modbases_dam-dcm-cpg_hac.cfg. The methylated sites are then detected and annotated using modPhred (Pryszcz and Novoa 2021) (https://github.com/novoalab/modPhred). For the purpose of creating unbiased DNA sequence logos modPhred was run to detect minimum modification frequencies of $0 \%$, to include all sites that were modified across all replicates. To compare methylation at frequencies between plasmids grown in WT vs dam $/ d \mathrm{~cm}^{-}$competent $E$. coli, we restricted analysis to sites with consensus methylation sequences.

Contamination - computational simulation of known plasmids

We simulated reads from established plasmids maps downloaded from Addgene. Our goal was to sample from commonly used plasmids, so we first took sequences from the Addgene 'top 15' list, with the remaining 84 randomly selected by traversing the Addgene 'Blue Flame Award' list alphabetically and selecting a single Blue Flame plasmid from each lab (https://blog.addgene.org/15-years-of-addgene-the-top15-plasmids) attempting to avoid any related plasmids. The full list of the simulated plasmids is in supplemental table 7 . The resulting plasmid lengths ranged from 2830 to 16973 bases, with a mean of 8080 . Plasmid sequences were downloaded and reads simulated using a version of BadRead customized to empirically draw read lengths from our established fragment lengths over a circular reference, using standard BadRead parameters for 'bad' reads (junk reads and chimeric reads) totaling $3 \% \quad(R$. Wick 2019) (https://github.com/aaronmck/Badread). Contamination was simulated for the five Addgene plasmids chosen as our controls (Addgene IDs 31815, 16337, 12251, 49792, and 52961) in five replicates each, representing relatively small to relatively large plasmids. These control plasmids were artificially contaminated with reads randomly drawn from all other plasmids at the following contamination rates: $0,1,2,3,4,5,6,7,8,9$, $10,15,20,25,30,40,50,60,70,80,90,95,98$, and $99 \%$.

Contamination is then estimated by first aligning Nanopore reads to a duplicated reference map of the plasmid (to capture reads spanning the circular breakpoint) using Minimap2 (https://github.com//h3/minimap2). Both aligned and unaligned reads are then assessed using a custom Python script. Our Bayesian formulation assumes a flat prior across equality spaced, discrete contamination level hypotheses, much like our approach in Cibulskis and McKenna et al. (Cibulskis et al. 2011):

$$
p(C \mid B, V, \epsilon)=\frac{p(B \mid C, V, \epsilon) * p(C))}{p(B))}
$$

Where $p(C)$ is our flat prior and the denominator $p(B)$ is the same over all contamination $(C)$ levels. We then need to evaluate the likelihood function. Assuming reads are independent and that our randomly-sampled bases from the read-alignment pair are independent, controling for read length, we can define the likelihood as a product of probabilities:

$p(B \mid C, V, \epsilon)=\prod_{i=1}^{N} \prod_{j=1}^{\left\|B_{N}\right\|} p\left(b_{i j} \mid c, \epsilon, v\right)$

With i...N representing the individual sequencing reads. We can define the following piecewise function:

$p\left(b_{i j} \mid c, b_{i k}, \epsilon, v\right)= \begin{cases}(1-c)(1-\epsilon)+c(1 / 4+v) & \text { if }\left(b_{i j}=b_{i k}\right) \\ (1-c)(\epsilon)+c(3 / 4-v) & \text { if }\left(b_{i j} \neq b_{i k}\right)\end{cases}$

Where $b_{i j}$ and $b_{i k}$ represent the corresponding aligned reference base $\mathrm{j}$ and sequencing base $\mathrm{k}$ when comparing read $\mathrm{i}$; $\mathrm{c}$ is the contamination rate, and epsilon is the Phred-scaled error rate. The parameter $v$ represents a constant shared sequence proportion between plasmids, estimated by comparing kmer proportions between all sampled addgene plasmid sequences (here set to 0.1984 ). Code to estimate this parameter is included in the GitHub repository. Contamination likelihoods are then normalized to 1 over the range $[0,1]$ and the mode of this distribution is found representing the maximum a posteriori (MAP) score. For simplicity in this paper we chose to sample over 100 bins from $[0,1]$, though increasing this resolution is trivial at the cost of computational time. 


\section{References}

Adey, Andrew C. 2021. "Tagmentation-Based SingleCell Genomics." Genome Research 31 (10): 16931705.

Adey, Andrew, Hilary G. Morrison, Asan, Xu Xun, Jacob O. Kitzman, Emily H. Turner, Bethany Stackhouse, et al. 2010. "Rapid, Low-Input, Low-Bias Construction of Shotgun Fragment Libraries by High-Density in Vitro Transposition." Genome Biology 11 (12): R119.

Amarasinghe, Shanika L., Shian Su, Xueyi Dong, Luke Zappia, Matthew E. Ritchie, and Quentin Gouil. 2020. "Opportunities and Challenges in Long-Read Sequencing Data Analysis." Genome Biology 21 (1): 30.

Antebi, Yaron E., James M. Linton, Heidi Klumpe, Bogdan Bintu, Mengsha Gong, Christina Su, Reed McCardell, and Michael B. Elowitz. 2017. "Combinatorial Signal Perception in the BMP Pathway." Cell 170 (6): 1184-1196.e24.

Buschmann, Tilo, and Leonid V. Bystrykh. 2013. "Levenshtein Error-Correcting Barcodes for Multiplexed DNA Sequencing." BMC Bioinformatics. https://doi.org/10.1186/1471-2105-14-272.

Cibulskis, Kristian, Aaron McKenna, Tim Fennell, Eric Banks, Mark DePristo, and Gad Getz. 2011. "ContEst: Estimating Cross-Contamination of Human Samples in next-Generation Sequencing Data." Bioinformatics 27 (18): 2601-2.

Currin, Andrew, Neil Swainston, Mark S. Dunstan, Adrian J. Jervis, Paul Mulherin, Christopher J. Robinson, Sandra Taylor, et al. 2019. "Highly Multiplexed, Fast and Accurate Nanopore Sequencing for Verification of Synthetic DNA Constructs and Sequence Libraries." Synthetic Biology 4 (1): ysz025.

De Coster, Wouter, Svenn D'Hert, Darrin T. Schultz, Marc Cruts, and Christine Van Broeckhoven. 2018. "NanoPack: Visualizing and Processing Long-Read Sequencing Data." Bioinformatics 34 (15): 2666-69.

Di Tommaso, Paolo, Maria Chatzou, Evan W. Floden, Pablo Prieto Barja, Emilio Palumbo, and Cedric Notredame. 2017. "Nextflow Enables Reproducible Computational Workflows." Nature Biotechnology 35 (4): 316-19.

Ewing, B., L. Hillier, M. C. Wendl, and P. Green. 1998. "Base-Calling of Automated Sequencer Traces Using Phred. I. Accuracy Assessment." Genome Research 8 (3): 175-85.

Gallegos, Jenna E., Mark F. Rogers, Charlotte A. Cialek, and Jean Peccoud. 2020. "Rapid, Robust Plasmid Verification by de Novo Assembly of Short Sequencing Reads." Nucleic Acids Research 48 (18): e106.

Gibson, Daniel G., Lei Young, Ray-Yuan Chuang, J. Craig Venter, Clyde A. Hutchison 3rd, and Hamilton O. Smith. 2009. "Enzymatic Assembly of DNA
Molecules up to Several Hundred Kilobases." Nature Methods 6 (5): 343-45.

Hawkins, John A., Stephen K. Jones Jr, llya J. Finkelstein, and William H. Press. 2018. "IndelCorrecting DNA Barcodes for High-Throughput Sequencing." Proceedings of the National Academy of Sciences of the United States of America 115 (27): E6217-26.

Heather, James M., and Benjamin Chain. 2016. "The Sequence of Sequencers: The History of Sequencing DNA." Genomics 107 (1): 1-8.

Hennig, Bianca P., Lars Velten, Ines Racke, Chelsea Szu Tu, Matthias Thoms, Vladimir Rybin, Hüseyin Besir, Kim Remans, and Lars M. Steinmetz. 2018. "Large-Scale Low-Cost NGS Library Preparation Using a Robust Tn5 Purification and Tagmentation Protocol." G3 8 (1): 79-89.

Hernandez-Lopez, Rogelio A., Wei Yu, Katelyn A. Cabral, Olivia A. Creasey, Maria Del Pilar Lopez Pazmino, Yurie Tonai, Arsenia De Guzman, et al. 2021. "T Cell Circuits That Sense Antigen Density with an Ultrasensitive Threshold." Science 371 (6534): 1166-71.

Hughes, Randall A., and Andrew D. Ellington. 2017. "Synthetic DNA Synthesis and Assembly: Putting the Synthetic in Synthetic Biology." Cold Spring Harbor Perspectives in Biology 9 (1). https://doi.org/10.1101/cshperspect.a023812.

Jun, Goo, Matthew Flickinger, Kurt N. Hetrick, Jane M. Romm, Kimberly F. Doheny, Gonçalo R. Abecasis, Michael Boehnke, and Hyun Min Kang. 2012. "Detecting and Estimating Contamination of Human DNA Samples in Sequencing and Array-Based Genotype Data." American Journal of Human Genetics 91 (5): 839-48.

Kieleczawa, J., J. J. Dunn, and F. W. Studier. 1992. "DNA Sequencing by Primer Walking with Strings of Contiguous Hexamers." Science 258 (5089): 178791.

Kolek, Jan, Karel Sedlar, Ivo Provaznik, and Petra Patakova. 2016. "Dam and Dcm Methylations Prevent Gene Transfer into Clostridium Pasteurianum NRRL B-598: Development of Methods for Electrotransformation, Conjugation, and Sonoporation." Biotechnology for Biofuels 9 (January): 14.

Kolmogorov, Mikhail, Jeffrey Yuan, Yu Lin, and Pavel A. Pevzner. n.d. "Assembly of Long Error-Prone Reads Using Repeat Graphs." https://doi.org/10.1101/247148.

Koren, Sergey, Brian P. Walenz, Konstantin Berlin, Jason R. Miller, Nicholas H. Bergman, and Adam M. Phillippy. 2017. "Canu: Scalable and Accurate LongRead Assembly via Adaptive k-Mer Weighting and Repeat Separation." Genome Research 27 (5): 72236.

Lunter, Gerton, and Martin Goodson. 2011. "Stampy: A Statistical Algorithm for Sensitive and Fast Mapping 
of Illumina Sequence Reads." Genome Research 21 (6): 936-39.

Marinus, M. G., and A. Løbner-Olesen. 2014. "DNA Methylation." EcoSal Plus 6 (1). https://doi.org/10.1128/ecosalplus.ESP-0003-2013.

New England Biolabs. n.d. "Making Unmethylated (DamDcm-) DNA." Accessed November 18, 2021. https://www.neb.com/tools-and-resources/usageguidelines/making-unmethylated-dam-dcm-dna.

Nichol, Kerrie, and Christopher E. Pearson. 2002. "CpG Methylation Modifies the Genetic Stability of Cloned Repeat Sequences." Genome Research 12 (8): 1246-56.

Picelli, Simone, Asa K. Björklund, Björn Reinius, Sven Sagasser, Gösta Winberg, and Rickard Sandberg. 2014. "Tn5 Transposase and Tagmentation Procedures for Massively Scaled Sequencing Projects." Genome Research 24 (12): 2033-40.

Pryszcz, Leszek P., and Eva Maria Novoa. 2021. "ModPhred: An Integrative Toolkit for the Analysis and Storage of Nanopore Sequencing DNA and RNA Modification Data." Bioinformatics, July. https://doi.org/10.1093/bioinformatics/btab539.

Reznikoff, William S. 2008. "Transposon Tn5." Annual Review of Genetics 42: 269-86.

Sanger, F., S. Nicklen, and A. R. Coulson. 1977. "DNA Sequencing with Chain-Terminating Inhibitors." Proceedings of the National Academy of Sciences of the United States of America 74 (12): 5463-67.

Shapland, Elaine B., Victor Holmes, Christopher D. Reeves, Elena Sorokin, Maxime Durot, Darren Platt, Christopher Allen, et al. 2015. "Low-Cost, HighThroughput Sequencing of DNA Assemblies Using a Highly Multiplexed Nextera Process." ACS Synthetic Biology 4 (7): 860-66.

Shendure, Jay, Shankar Balasubramanian, George M. Church, Walter Gilbert, Jane Rogers, Jeffery A. Schloss, and Robert H. Waterston. 2017. "DNA Sequencing at 40: Past, Present and Future." Nature 550 (7676): 345-53.

Wick, Ryan. 2019. "Badread: Simulation of Error-Prone Long Reads." Journal of Open Source Software 4 (36): 1316.

Wick, Ryan R., and Kathryn E. Holt. 2019. "Benchmarking of Long-Read Assemblers for Prokaryote Whole Genome Sequencing." F1000Research 8 (December): 2138.

Wick, Ryan R., Louise M. Judd, Claire L. Gorrie, and Kathryn E. Holt. 2017. "Completing Bacterial Genome Assemblies with Multiplex MinION Sequencing." Microbial Genomics 3 (10): e000132. 
bioRxiv preprint doi: https://doi.org/10.1101/2022.01.25.477550; this version posted January 26, 2022. The copyright holder for this preprint (which was not certified by peer review) is the author/funder, who has granted bioRxiv a license to display the preprint in perpetuity. It is made available under aCC-BY-NC-ND 4.0 International license.

\section{Supplementary Figures}

A

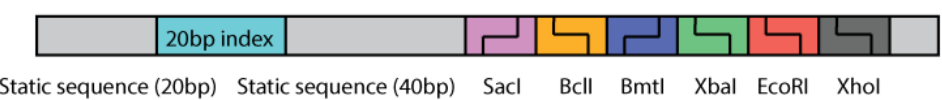

B

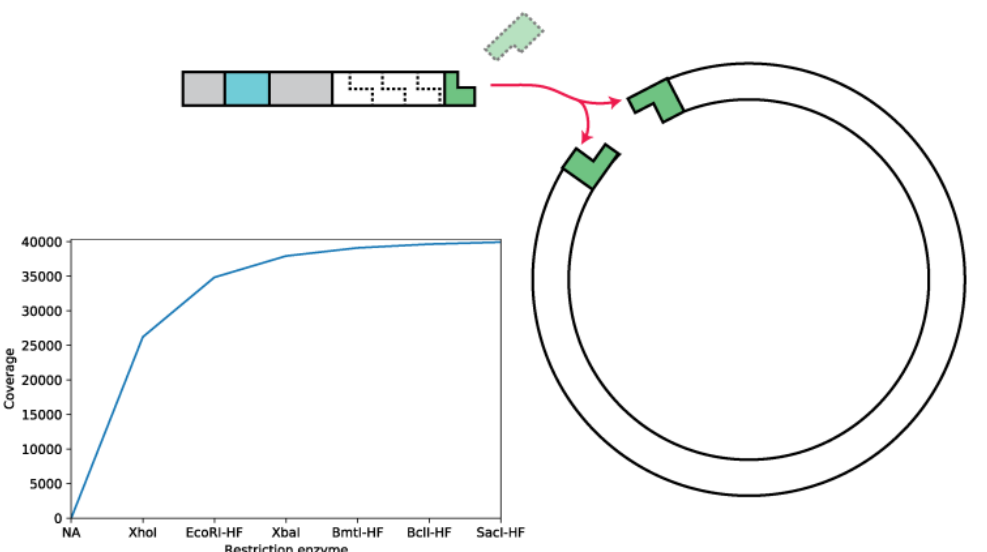

Sup. figure 1. (A). Barcode ligation requires a single, known restriction site (green) for each plasmid that is shared with the ligation adapters. Using a set cover approach, we searched all known plasmids in the Addgene database to establish a candidate of restriction enzymes that cut once and only once per plasmid (see methods). From this search, we chose a set of 6 restriction enzymes that were incorporated into unique tagged barcodes for ligation. (B) These restriction enzymes covered a total of $62 \%$ of the 63,017 Addgene plasmids with a single restriction site. Although this approach offers a successful and streamlined approach to barcoding our hope was to be agnostic to the sequence of each plasmid. 
bioRxiv preprint doi: https://doi.org/10.1101/2022.01.25.477550; this version posted January $26,2022$. The copyright holder for this preprint (which was not certified by peer review) is the author/funder, who has granted bioRxiv a license to display the preprint in perpetuity. It is made available under aCC-BY-NC-ND 4.0 International license.

A
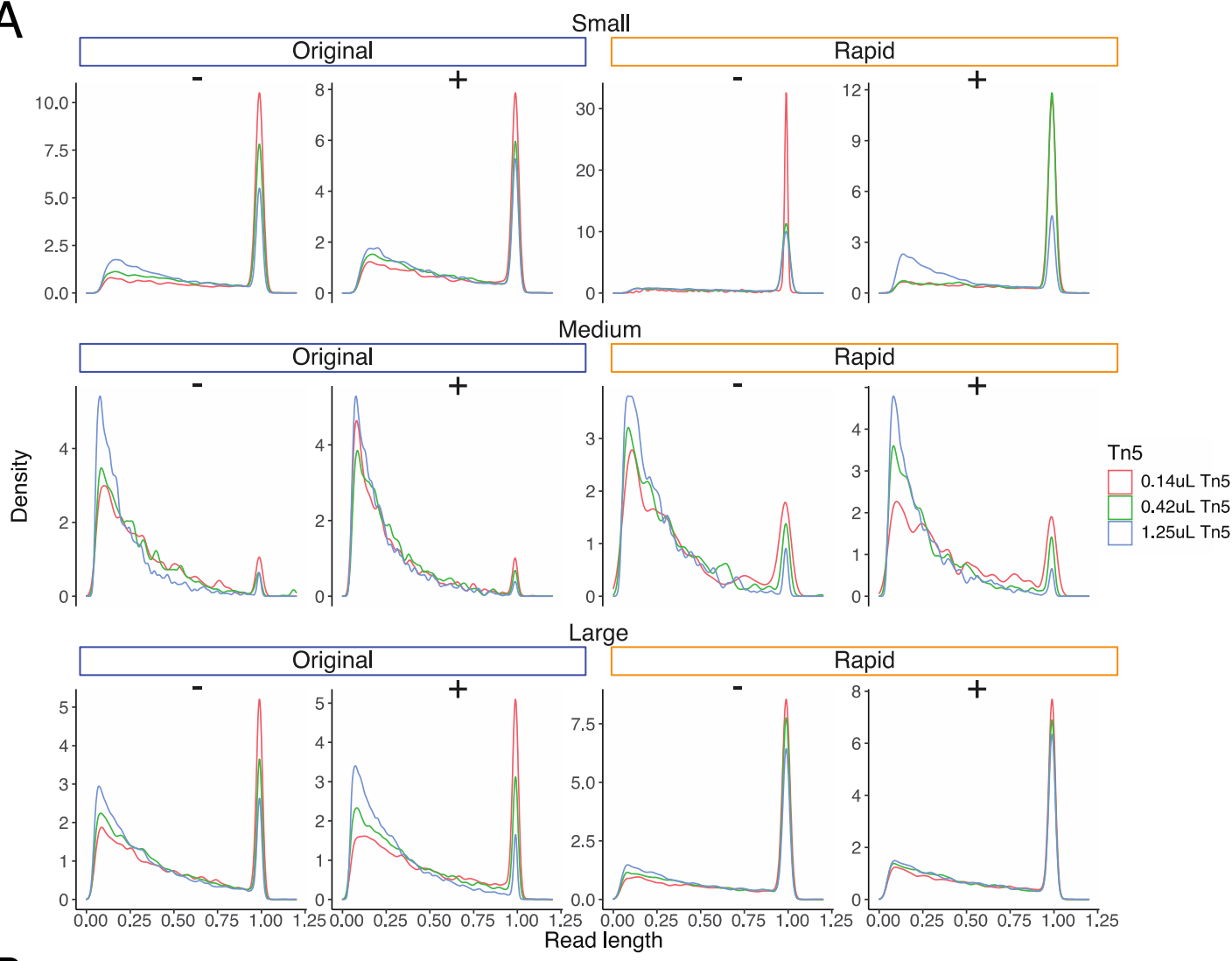

$\mathrm{B}$

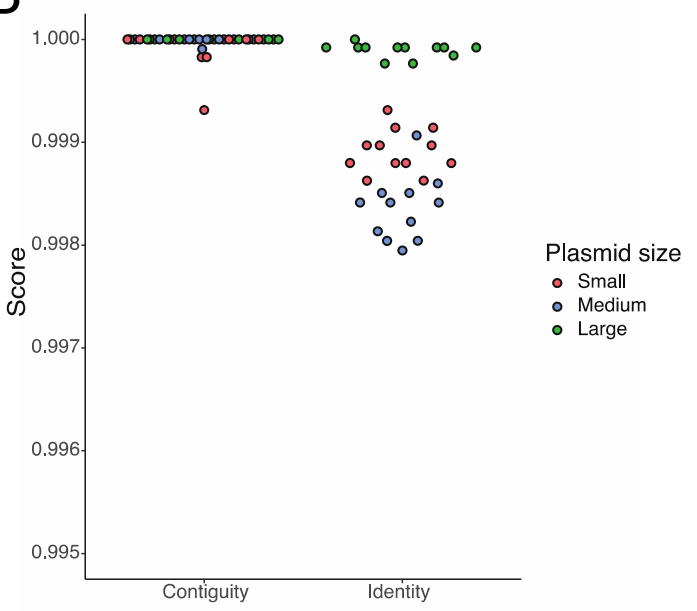

Sup. figure 2. (A). To determine conditions that would maximize read length we processed three plasmids of various lengths (6kbp to $13 \mathrm{kbp}$ ) through a modified pipeline. Density plots of read lengths, normalized to the full length plasmid, are shown under the conditions of changing length (top to bottom), amount of Tn5 (colors), the duration of tagmentation (left and right), as well as method for inactivation (- for SDS only, + for SDS with heat inactivation). (B). Once the reads from each of the conditions were assembled we calculated contiguity and identity scores for each assembly. Identity scores cluster by plasmid type due to sequence complexity and are unrelated to reaction conditions. 
bioRxiv preprint doi: https://doi.org/10.1101/2022.01.25.477550; this version posted January $26,2022$. The copyright holder for this preprint (which was not certified by peer review) is the author/funder, who has granted bioRxiv a license to display the preprint in perpetuity. It is made available under aCC-BY-NC-ND 4.0 International license.

A

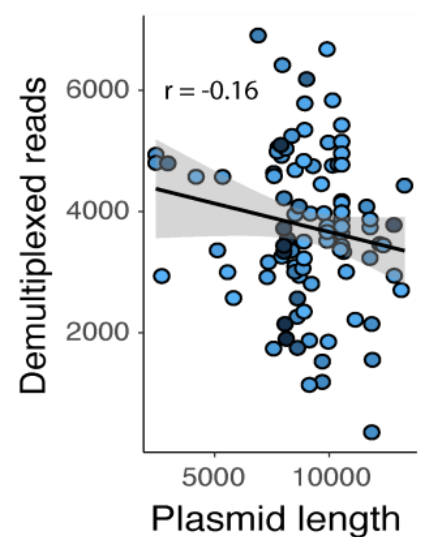

C

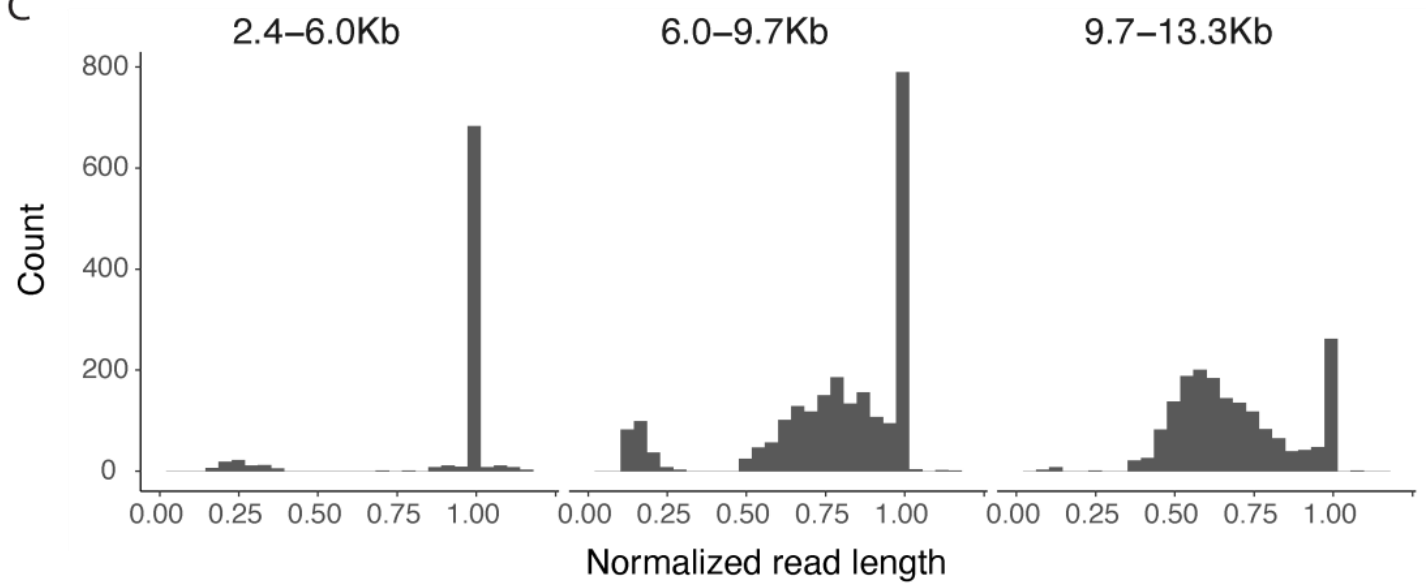

D

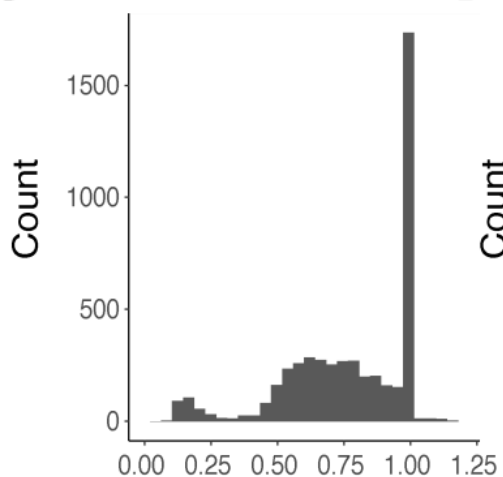

Normalized read length
E

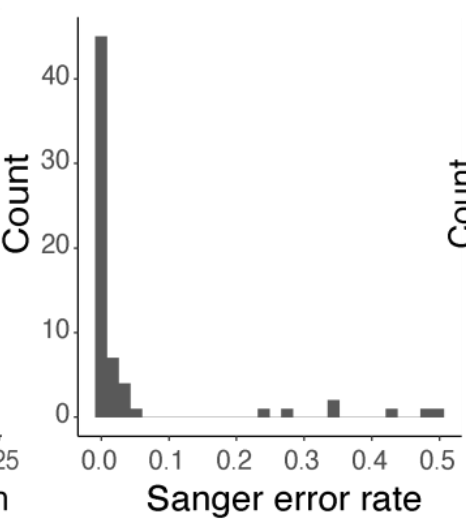

B

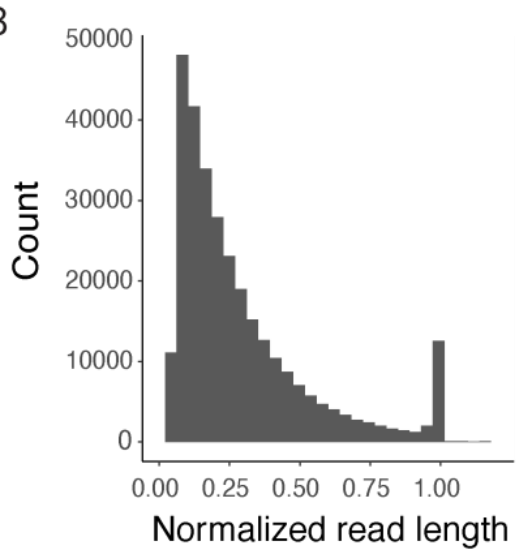

Sup. figure 3. (A). The correlation of recovered Nanopore sequencing read counts to input plasmid length, where point color indicates the relative repeat content of the input plasmid. (B) Histogram of unfiltered Nanopore read lengths normalized to the originating plasmid sequence length. (C) Post-filtering, normalized read lengths across plasmid lengths and (D) the global distribution. (E) Error rates for all 64 Sanger sequencing trace files after quality control and (F) after removing 7 traces with error rates over $10 \%$. 
bioRxiv preprint doi: https://doi.org/10.1101/2022.01.25.477550; this version posted January 26, 2022. The copyright holder for this preprint (which was not certified by peer review) is the author/funder, who has granted bioRxiv a license to display the preprint in perpetuity. It is made available under aCC-BY-NC-ND 4.0 International license.
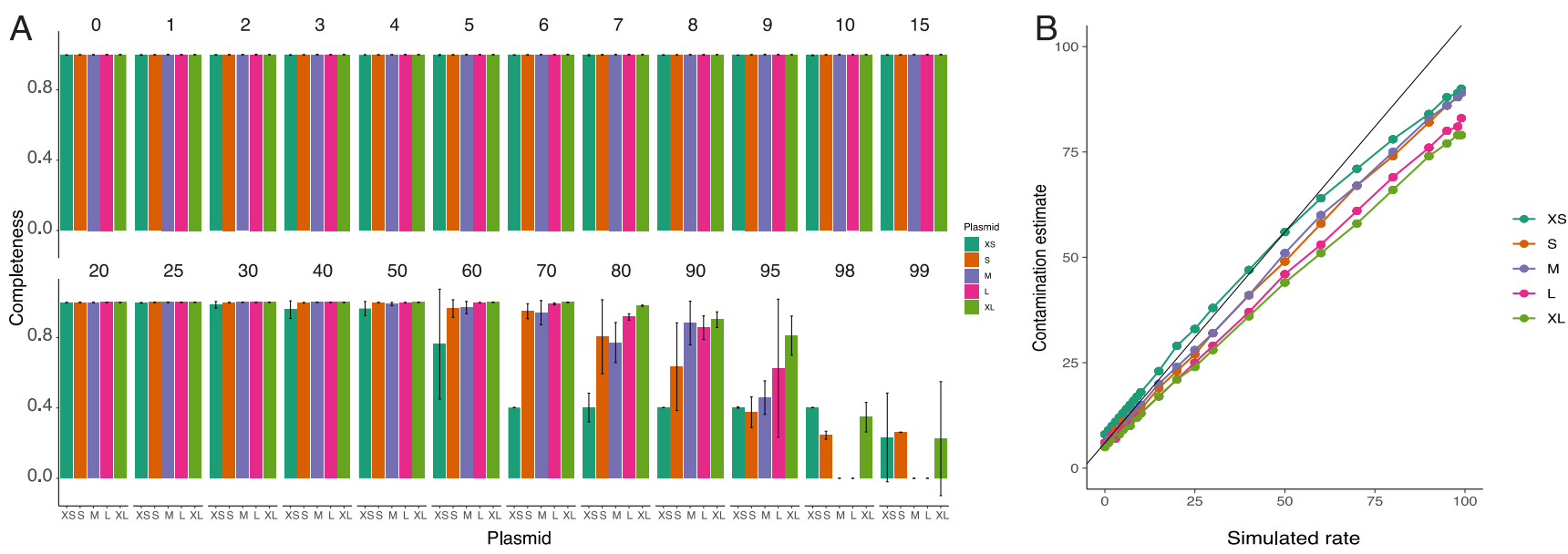

Supplemental figure 4. (A) Plasmid assembly completeness from simulated reads. Each of five plasmids (XS=extra small, $S=s m a l l$, $\mathrm{M}=$ medium, $\mathrm{L}=$ large, $\mathrm{XL}=$ extra large) were mixed with randomly sampled reads from the remaining 91 plasmids at contamination levels from $0 \%$ to $99 \%$. Assembly issues in the smaller plasmids started at $30-40 \%$ contaminated, with most assemblies having serious issues at contamination levels greater than $80 \%$. (B) Contamination estimates when reads are mapped against the known plasmid map instead of the assembled reference as in figure 3 . Here contamination estimates better track simulated rates to the upper limits of contamination. 